\title{
Cooperative Control Design for Time-Varying Formations of Multi-Agent Systems
}

\author{
Lara Briñón-Arranz, Alexandre Seuret, and Carlos Canudas-de-Wit
}

\begin{abstract}
This paper deals with cooperative control design for nonlinear multi-agent systems. The control objective is to ensure that a group of agents reaches a formation characterized by external time-varying parameters. Firstly, a translation control design is presented to stabilize the multi-agent system to a circular motion tracking a time-varying center. Then, we propose a new framework based on affine transformations to extend previous results to more complex time-varying formations. Moreover, both control laws are improved adding a cooperative term to distribute the agents uniformly along the formation.
\end{abstract}

\section{INTRODUCTION}

Cooperative control problems and multi-agent systems have received much attention in recent years. The field includes consensus algorithms for multi-agent systems [1], [2], distributed sensor networks [3] and autonomous systems as autonomous underwater and unmanned air vehicles (AUVs and UAVs) [4], [5]. Formation control is an important issue in coordinated control for multi-agent systems. A formation is defined as a group of autonomous agents with communication capacities, forming a particular configuration (i.e., desired positions and orientations), in which the agents collaborate to achieve a common goal [6]. A particular class of motion coordination for multi-agent systems considering several constraints is studied in [3], [7]. These previous works studied the problem of design feedback control laws that stabilize a set of agents to circular and parallel formations. These interesting results deal with time-invariant circular formations which have a fixed center and a constant radius. Some extensions

L. Briñón-Arranz is with the Laboratory of Robotics and Systems in Engineering and Science (LARSyS), Instituto Superior Técnico (IST), University of Lisbon, Portugal. A. Seuret is with the CNRS, LAAS, 7 avenue du colonel Roche, 2 Univ de Toulouse, F-31400 Toulouse, France. C. Canudas-de-Wit is with the CNRS, GIPSA-lab, Grenoble, France. E-mail: lara.arranz@ist.utl.pt, alexandre.seuret@laas.fr, carlos.canudas-de-wit@gipsa-lab.grenoble-inp.fr. 
based on these works have been developed: three-dimensional motion coordination in a flow-field [8] and stabilization of a fleet to fixed convex forms [9], for instance.

A relevant problem is to relax these constraints and to consider time-varying formations, i.e. allowing the center and radius of a circular formation to be time-varying. Indeed, time-varying configurations are appropriate to some applications where the agents perform collaborative tasks requiring the formation to displace towards an a priori unknown direction and to adapt to some particular form. For instance, in source seeking applications, the formation is driven following the source gradient direction [4], [10]. Target tracking problems also require to consider time-varying formations. Some cooperative approaches to achieve this challenge using a fleet of vehicles have been studied in the literature [11]. A circular formation encircling a time-varying target seems appropriate to approach target tracking problems, see [12]. On the other side, changing the shape of a formation can be useful in several situations. For instance, it can be seen as a collision avoidance method such that, a circular formation of vehicles reduces its radius in order to go through a narrow place.

In both recent papers [13], [14] a vector field control approach is presented in order to enforce a simple integrator vehicle to converge to time-varying target curves. In this paper, we propose novel cooperative control laws to stabilize a fleet of vehicles to large classes of time-varying formations considering a unicycle model for the dynamics. Firstly, based on previous circular formation control results studied in literature [3], [7], [15], a new control design to make the vehicles converge to a circular motion following a time-varying reference of its center is provided. A new control strategy using an autonomous stable exosystem and a tracking control design is proposed. Contrary to our previous work [16], the resulting control law does not depend on initial agents' states. The main contribution of this paper is to generalize this control law to larger class of formations, not only circular. Using the three affine transformations (rotation, translation and scaling) a general time-varying formation control law will be expressed in a generic and compact way. An additional feature of the control adds the possibility to distribute uniformly the agents along the formation in a collaborative way.

\section{Problem Formulation}

\section{A. Model of the agents}

Consider a group of $N$ identical vehicles modeled with unicycle kinematics subject to a simple nonholonomic constraint such that, the dynamics of agent $k=1, \ldots, N$ is defined by: 


$$
\begin{aligned}
\dot{\mathbf{r}}_{k} & =v_{k}\left[\cos \theta_{k} \sin \theta_{k}\right]^{T} \\
\dot{\theta}_{k} & =u_{k}
\end{aligned}
$$

where $\mathbf{r}_{k} \in \mathbb{R}^{2}$ is the position vector of agent $k, \theta_{k}$ is its heading angle and $v_{k}, u_{k}$ are the control inputs.

\section{B. Exosystem}

Our approach is based on the previous results exposed in [3], [7] to obtain time-invariant circular formations and the beacon control law from [15] dealing with circular motions. In these works, the vehicles are represented by (1) with unit linear velocity, i.e. $v_{k}=1, \forall k$. In order to exploit these previous results, we introduce an exosystem represented by the following multi-agent dynamics:

$$
\begin{aligned}
\dot{\hat{\mathbf{r}}}_{k} & =v_{0}\left[\cos \psi_{k} \sin \psi_{k}\right]^{T} \\
\dot{\psi}_{k} & =\hat{u}_{k}
\end{aligned}
$$

where $v_{0}>0, \psi_{k}$ represents the angular orientation of the velocity vector $\dot{\hat{\mathbf{r}}}_{k}$ and $\hat{u}_{k}$ is the control input. We can design different control laws $\hat{u}_{k}$ in order to obtain an autonomous stable exosystem. For instance, the circular control law presented in [15], [7] defined by following equation

$$
\hat{u}_{k}=\omega_{0}\left(1+\kappa \dot{\hat{\mathbf{r}}}_{k}^{T} \hat{\mathbf{r}}_{k}\right)-\frac{\partial U}{\partial \psi_{k}}
$$

ensures that the exosystem represented by (2) is stabilized to a circular motion centered at the origin of the coordinates frame and with radius $R=v_{0} /\left|\omega_{0}\right|$, where $\omega_{0} \neq 0$ is the angular velocity. Moreover, the agents of the exosystem are uniformly distributed due to the cooperative term $U(\psi)$ which includes information of others agents, as shown in Fig. 1. The communication protocol assumed in this paper depends on the distances between the vehicles. This collaborative approach will be discussed in Section IV. The exosystem can be used to generate the desired trajectories for the multi-agent system (1), see Fig. 1. The main advantage of using an autonomous exosystem is that elegant and effective distributed control laws can be easily applied to the multi-agent dynamics (2).

\section{Control Objectives}

The following assumptions are considered in the sequel:

- Each vehicle knows its absolute vector position $\mathbf{r}_{k}$ with respect to the global inertial frame.

- The time-varying references which define the parameters of the formation (e.g. the center and radius of a circular formation) are known to all the vehicles. 


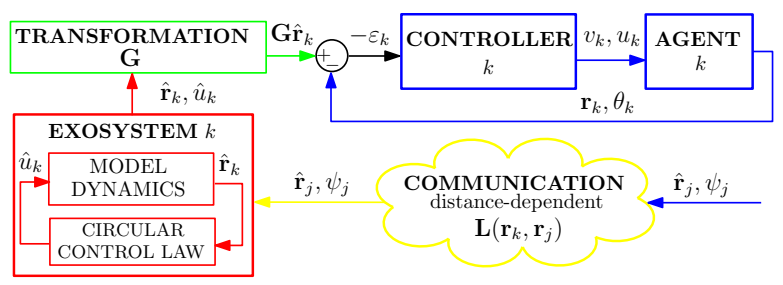

Fig. 1. Structure for the feedback design including a decentralized exosystem. Each agent $k$ computes its own exosystem which communicates with other exosystems by means of a communication protocol depending on the distance between the agents (the Laplacian matrix $\mathbf{L}$ depends on the agents' states $\mathbf{r}_{k}$ ).

Under these assumptions, the main objective is to design control laws $\left(v_{k}, u_{k}\right)$ such that the fleet of agents represented by system (1) converges to a particular formation defined by a few number of time-varying given parameters. Moreover, we consider a cooperative approach to distribute the vehicles in a desired pattern along the formation.

Several time-varying formations can be obtained deforming a unit circle, as we will present in Section $\mathrm{V}$. The main idea here is thus to deform a circular formation applying a general transformation G composed of rotations, translations and scaling, which will be mathematically defined in Section V. For instance, an ellipsis results from applying a non-uniform scaling to the unit circle. Thanks to this transformation $\mathbf{G}$, the desired trajectories of the agents are defined by a few number of parameters which could be time-varying. Our idea is to transform the exosystem circular trajectories via a transformation $\mathbf{G}$ in order to design new time-varying curves. The resulting transformed trajectories will be considered as a reference to the multi-agent states, see Fig. 1. The error between the reference and the multi-agent state is defined by:

$$
\varepsilon_{k}=\mathbf{r}_{k}-\mathbf{G} \hat{\mathbf{r}}_{k}, \quad \forall k=1, \ldots, N
$$

Our problem is to design control laws $\left(v_{k}, u_{k}\right)$ for the multi-agent system (1) such that the error $\varepsilon_{k}$ converges to zero.

\section{TRANSLATION OF A CIRCULAR MOTION}

Firstly, in order to stabilize a fleet of agents to a time-varying circular motion, we propose a new control strategy. This new strategy follows three phases:

Transformation: the desired formation is expressed as result of a sequence of transformations applied to the unit circle and defined by a few number of parameters. 
Exosystem: we define a global stable exosystem (2), for instance, stabilized to a circular motion with fixed center thanks to the beacon control law from [15].

Tracking control design: we design the control inputs $\left(v_{k}, u_{k}\right)$ of the multi-agent system (1) in order to track the reference defined by the transformed circular trajectories of the exosystem, i.e., enforcing the error (4) to converge to zero.

\section{A. Transformation}

As a first step, we present the case of a circular motion tracking a time-varying center $\mathbf{c}(t)$. The transformation applied in this case to the exosystem is a translation by a vector $\mathbf{c}(t) \in \mathbb{R}^{2}$. In this situation, the desired trajectories are defined by the position vector of the exosystem and the external parameter c. Therefore, the error becomes:

$$
\varepsilon_{k}=\mathbf{r}_{k}-\left(\hat{\mathbf{r}}_{k}+\mathbf{c}\right) \quad \forall k=1, \ldots, N
$$

\section{B. Design a global stable exosystem}

The exosystem will be stabilized to converge to a circular motion with radius $R$. According to the relation between linear and angular velocities satisfied during a circular motion, the linear velocity is imposed to be $v_{0}=R\left|\omega_{0}\right|$. In this section no collaborative approach is considered, consequently the control input for the exosystem does not include cooperative terms. The circular control law (3) with $U(\psi)=0$ enforces the exosystem defined by (2) to converge to a circular motion. In order to clarify this result, we present here a sketch of the proof. Consider the notation $\hat{\mathbf{r}}=\left(\hat{\mathbf{r}}_{1}^{T}, \ldots, \hat{\mathbf{r}}_{N}^{T}\right)^{T}$, $\psi=\left(\psi_{1}, \ldots, \psi_{N}\right)^{T}$, and the following Lyapunov function

$$
S(\hat{\mathbf{r}}, \psi)=\frac{1}{2} \sum_{k=1}^{N}\left\|\dot{\hat{\mathbf{r}}}_{k}-\omega_{0} \mathbf{R}_{\frac{\pi}{2}} \hat{\mathbf{r}}_{k}\right\|^{2} \geq 0
$$

where $\mathbf{R}_{\frac{\pi}{2}}=\left[\begin{array}{cc}0 & -1 \\ 1 & 0\end{array}\right]$ denotes a rotation matrix through an angle $\frac{\pi}{2}$ counterclockwise around the origin. At the equilibrium points of the previous Lyapunov function, i.e., $S(\hat{\mathbf{r}}, \psi)=0$, the dynamics of the exosystem (2) satisfies $\dot{\mathbf{r}}_{k}-\omega_{0} \mathbf{R}_{\frac{\pi}{2}} \hat{\mathbf{r}}_{k}=0$. Thus, the position vector and its velocity vector are perpendicular, i.e., $\dot{\hat{\mathbf{r}}}_{k}^{T} \hat{\mathbf{r}}_{k}=0$. This condition leads to the kinematic relation for the rotation of a rigid body. In other words, the vectors $\hat{\mathbf{r}}_{k}$ are turning around the frame origin at the equilibrium. Considering the proposed control law (3) with $U(\psi)=0$ and evaluating the derivative of $S(\hat{\mathbf{r}}, \psi)$ along the solutions of the resulting closed-loop system (2) leads to: 


$$
\begin{aligned}
\dot{S}(\hat{\mathbf{r}}, \psi) & =\sum_{k=1}^{N}\left(\hat{u}_{k} \mathbf{R}_{\frac{\pi}{2}} \dot{\hat{\mathbf{r}}}_{k}-\omega_{0} \mathbf{R}_{\frac{\pi}{2}} \dot{\hat{\mathbf{r}}}_{k}\right)^{T}\left(\dot{\hat{\mathbf{r}}}_{k}-\omega_{0} \mathbf{R}_{\frac{\pi}{2}} \hat{\mathbf{r}}_{k}\right) \\
& =\sum_{k=1}^{N} \omega_{0} \hat{\mathbf{r}}_{k}^{T} \dot{\hat{\mathbf{r}}}_{k}\left(\omega_{0}-\hat{u}_{k}\right)=-\kappa \sum_{k=1}^{N}\left(\omega_{0} \hat{\mathbf{r}}_{k}^{T} \dot{\hat{\mathbf{r}}}_{k}\right)^{2} \leq 0 .
\end{aligned}
$$

In conclusion, $S(\hat{\mathbf{r}}, \psi)$ is a suitable Lyapunov function for the exosystem (2), and by the LaSalle Invariance Principle the solutions converge to the largest invariant set $\Lambda$, for which $\dot{S}=0$. Consequently, the dynamics of the exosystem satisfies $\dot{\hat{\mathbf{r}}}_{k}=\omega_{0} \mathbf{R}_{\frac{\pi}{2}} \hat{\mathbf{r}}_{k}$ which corresponds to a circular motion.

\section{Translation control design}

In order to enforce the convergence of the multi-agent system to the transformed trajectories generated by the exosystem, a tracking control design is proposed. The following theorem presents the first contribution of this paper.

Theorem 1 Consider a twice differentiable function $c(t): \mathbb{R} \rightarrow \mathbb{R}^{2}$, with bounded first and second timederivatives. Let $\omega_{0} \neq 0, \kappa>0, \alpha>0, \beta \geq 2 \sqrt{\alpha}$ be four control parameters, $R>0$ be the radius of the desired circular motion and the following condition is satisfied:

$$
\|\dot{\boldsymbol{c}}(t)\|<R\left|\omega_{0}\right| \quad \forall t
$$

Then, for all initial conditions $\boldsymbol{r}(0), \theta(0)$, the control law

$$
\begin{aligned}
& \dot{v}_{k}=-\beta v_{k}+\frac{\dot{\boldsymbol{r}}_{k}^{T}}{v_{k}}\left[\hat{u}_{k} \boldsymbol{R}_{\frac{\pi}{2}} \dot{\hat{\boldsymbol{r}}}_{k}+\ddot{\boldsymbol{c}}+\beta\left(\dot{\hat{\boldsymbol{r}}}_{k}+\dot{\boldsymbol{c}}\right)+\alpha\left(\hat{\boldsymbol{r}}_{k}+\boldsymbol{c}-\boldsymbol{r}_{k}\right)\right] \\
& u_{k}=\frac{\hat{u}_{k}}{v_{k}^{2}} \dot{\boldsymbol{r}}_{k}^{T} \dot{\hat{\boldsymbol{r}}}_{k}+\frac{\dot{\boldsymbol{r}}_{k}^{T} \boldsymbol{R}_{\frac{\pi}{2}}^{T}}{v_{k}^{2}}\left[\ddot{\boldsymbol{c}}+\beta\left(\dot{\hat{\boldsymbol{r}}}_{k}+\dot{\boldsymbol{c}}\right)+\alpha\left(\hat{\boldsymbol{r}}_{k}+\boldsymbol{c}-\boldsymbol{r}_{k}\right)\right]
\end{aligned}
$$

where $\dot{\hat{\boldsymbol{r}}}_{k}$ and $\hat{u}_{k}$ are respectively defined by (2) with $v_{0}=R\left|\omega_{0}\right|$ and (3) with $U(\psi)=0$, makes all the agents defined by (1) converge to a circular motion of radius $R$, and whose center tracks the time-varying reference $\boldsymbol{c}(t)$. The direction of rotation is determined by the sign of $\omega_{0}$.

Proof: The first step is to ensure the convergence of the exosystem defined by (2) to a fixed circular motion, as presented before. The second step corresponds to a tracking strategy. In order to achieve the objective $\mathbf{r}_{k} \rightarrow \hat{\mathbf{r}}_{k}+\mathbf{c}$, the tracking error is defined by (5). With a view to make the error converge to zero, we wish to impose the error dynamics $\ddot{\varepsilon}_{k}=-\beta \dot{\varepsilon}_{k}-\alpha \varepsilon_{k}$, where $\alpha>0, \beta \geq 2 \sqrt{\alpha}$. Thus, the error converges exponentially to zero. The dynamics of the error equation determines the control law for system (1) since:

$$
\ddot{\varepsilon}_{k}=\ddot{\mathbf{r}}_{k}-\ddot{\hat{\mathbf{r}}}_{k}-\ddot{\mathbf{c}}=\frac{\dot{v}_{k}}{v_{k}} \dot{\mathbf{r}}_{k}+u_{k} \mathbf{R}_{\frac{\pi}{2}} \dot{\mathbf{r}}_{k}-\hat{u}_{k} \mathbf{R}_{\frac{\pi}{2}} \dot{\mathbf{r}}_{k}-\ddot{\mathbf{c}}
$$


According to the error dynamics and multiplying this last equation by $\dot{\mathbf{r}}_{k}^{T}$ and by $\dot{\mathbf{r}}_{k}^{T} \mathbf{R}_{\frac{\pi}{2}}^{T}$, both following expressions hold:

$$
\begin{aligned}
& \dot{v}_{k} v_{k}=-\beta v_{k}^{2}+\dot{\mathbf{r}}_{k}^{T}\left[\hat{u}_{k} \mathbf{R}_{\frac{\pi}{2}} \dot{\hat{\mathbf{r}}}_{k}+\ddot{\mathbf{c}}+\beta\left(\dot{\hat{\mathbf{r}}}_{k}+\dot{\mathbf{c}}\right)+\alpha\left(\hat{\mathbf{r}}_{k}+\mathbf{c}-\mathbf{r}_{k}\right)\right] \\
& u_{k} v_{k}^{2}=\hat{u}_{k} \dot{\mathbf{r}}_{k}^{T} \dot{\hat{\mathbf{r}}}_{k}+\dot{\mathbf{r}}_{k}^{T} \mathbf{R}_{\frac{\pi}{2}}^{T}\left[\ddot{\mathbf{c}}+\beta\left(\dot{\hat{\mathbf{r}}}_{k}+\dot{\mathbf{c}}\right)+\alpha\left(\hat{\mathbf{r}}_{k}+\mathbf{c}-\mathbf{r}_{k}\right)\right] .
\end{aligned}
$$

By definition, this control law enforces exponential convergence of the tracking error dynamics away from the singularity $v_{k}=0$. Thanks to previous definition of the error when $t \rightarrow \infty, \mathbf{r}_{k}=\hat{\mathbf{r}}_{k}+\mathbf{c}$ and thus $\dot{\mathbf{r}}_{k}=\dot{\hat{\mathbf{r}}}_{k}+\dot{\mathbf{c}}, \forall k=1, \ldots, N$. Taking into account the circular control law (3), the exosystem converge to $\dot{\hat{\mathbf{r}}}_{k}=\omega_{0} \mathbf{R}_{\frac{\pi}{2}} \hat{\mathbf{r}}_{k}$, hence, the closed-loop dynamics of multi-agent system (1) describes a time-varying circular motion since for all $k=1, \ldots, N$ :

$$
\dot{\mathbf{r}}_{k}=\underbrace{\omega_{0} \mathbf{R}_{\frac{\pi}{2}}\left(\mathbf{r}_{k}-\mathbf{c}\right)}_{\text {circular motion }}+\underbrace{\dot{\mathbf{c}}}_{\text {translation }} .
$$

In this situation, the singular point $v_{k}=\left\|\dot{\mathbf{r}}_{k}\right\|=0$ is equivalent to $\left\|\omega_{0} \mathbf{R}_{\frac{\pi}{2}}\left(\mathbf{r}_{k}-\mathbf{c}\right)+\dot{\mathbf{c}}\right\|=0$ and can be rewritten as $\omega_{0} \mathbf{R}_{\frac{\pi}{2}}\left(\mathbf{r}_{k}-\mathbf{c}\right)=-\dot{\mathbf{c}}$. If condition (7) is satisfied then $\|\dot{\mathbf{c}}(t)\| \neq\left\|\omega_{0} \mathbf{R}_{\frac{\pi}{2}}\left(\mathbf{r}_{k}-\mathbf{c}\right)\right\|, \forall t$. Therefore, the singular point $v_{k}=0$ is avoided and the control inputs (8) are respectively obtained.

It is important to stress that this control law is robust to uncertainties in the initial conditions of the agents' states $\mathbf{r}_{k}(0), \theta_{k}(0)$ which represents the main difference with respect to our previous work [16].

\section{UNIFORM DISTRIBUTION ALONG A CIRCULAR FORMATION}

The previous control law does not take into consideration communication between agents and each vehicle converges independently to the desired circular motion. Therefore, the phase arrangement of the particles is arbitrary. In other words, to stabilize the agents to a circular formation, the translation control law must include a cooperative term to distribute the agents along the same circle following a particular pattern.

\section{A. Communication topology}

Let $\mathcal{G}=(V, E)$ be an undirected graph and $\mathbf{L}$ its Laplacian matrix. The set of agents is denoted by $V=\{1, \ldots, N\}, \mathcal{N}_{k}=\{j \in V:(k, j) \in E\}$ represents the set of neighbors of agent $k$ and $\left|\mathcal{N}_{k}\right|$ denotes its number of neighbors. In the sequel, $\otimes$ denotes the Kronecker product and, for simplicity, we define $\overline{\mathbf{L}}=\mathbf{L} \otimes \mathbf{I}_{2}$ where $\mathbf{I}_{N} \in \mathbb{R}^{N \times N}$ is the identity matrix. 


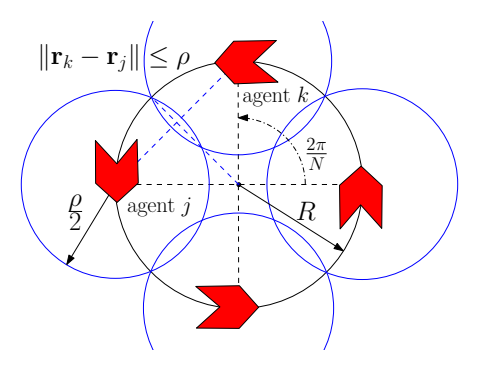

Fig. 2. Geometrical condition to assure a circulant communication graph for a group of agents in a circular formation with communication radius $\rho$.

Based on the ideas from synchronization of coupled oscillators, the authors from [7] introduced a potential function depending on the relative headings which induces a repulsion force to enlarge the angular distance between two connected agents in the circle

$$
U(\psi)=\frac{K}{N} \sum_{m=1}^{\lfloor N / 2\rfloor} \frac{1}{2 m^{2}} \mathbf{B}_{m}^{T} \overline{\mathbf{L}} \mathbf{B}_{m},
$$

where $\mathbf{B}_{m}=\left(\cos m \psi_{1}, \sin m \psi_{1}, \ldots, \cos m \psi_{N}, \sin m \psi_{N}\right)^{T}$ and $\lfloor N / 2\rfloor$ is the largest integer less than or equal to $N / 2$. The splay state corresponding to the uniform distribution of the agents along the circle is a critical point of $U(\psi)$. Applying this method to the translation control law is straightforward in the case of fixed communication graph. Nevertheless, the uniform distribution is only locally stable for fixed circulant graphs, see [7], and others configurations could be stabilized depending on the initial conditions. Moreover, considering fixed communication is not realistic because the distance between two linked agents is not considered, [1], [2]. Therefore, it might be more interesting to consider distancedependent communication graphs. This means that each agent can only receive information from its closed neighbors. In this situation, a communication area for each vehicle is introduced, defined by the critical communication distance $\rho$, see Fig. 2, which depends on the characteristics of the communication devices and the environment of the agents. The distance-dependent communication graph (also called proximity graph in literature [17]) is now time-varying because the position of vehicles is changing in time. Based on graph theory, the distance-dependent Laplacian matrix $\mathbf{L}(\mathbf{r}(t))$ is defined as follows:

$$
\mathbf{L}_{k, j}= \begin{cases}\left|\mathcal{N}_{k}\right|, & \text { if } \quad k=j \\ -1, & \text { if } \quad\left\|\mathbf{r}_{k}-\mathbf{r}_{j}\right\| \leq \rho \\ 0 & \text { otherwise }\end{cases}
$$




\section{B. Uniform Distribution Control Design}

The cooperative translation control for the distance-dependent communication assumption is presented in the following corollary:

Corollary 1 Consider a twice differentiable function $c(t): \mathbb{R} \rightarrow \mathbb{R}^{2}$, with bounded first and second time-derivatives. Let $\omega_{0} \neq 0, \kappa>0, \alpha>0, \beta \geq 2 \sqrt{\alpha}$ and $K>0$ be five control parameters, $R>0$ be the radius of the desired circular motion and condition (7) is satisfied. Let $\mathcal{G}(t)$ be the communication graph, $\boldsymbol{L}$ be its corresponding Laplacian matrix defined by (10) and the critical communication distance $\rho$ satisfies:

$$
\rho>2 R \sin \frac{\pi}{N}
$$

Then the control law (8) where $\dot{\hat{\boldsymbol{r}}}_{k}$ is defined by (2) with

$$
\left\{\begin{array}{l}
\hat{u}_{k}=\omega_{0}\left(1+\kappa \dot{\hat{\boldsymbol{r}}}_{k}^{T} \hat{\boldsymbol{r}}_{k}\right)-\frac{\partial U}{\partial \psi_{k}} \\
\frac{\partial U}{\partial \psi_{k}}=-\frac{K}{\left|\mathcal{N}_{k}\right|} \sum_{j \in \mathcal{N}_{k}} \sum_{m=1}^{\lfloor N / 2\rfloor} \frac{\sin m \psi_{k j}}{m}
\end{array}\right.
$$

where $\psi_{k j}=\psi_{k}-\psi_{j}$, makes all the agents defined by (1) converge to a circular formation of radius $R$ and whose center tracks the time-varying reference $\boldsymbol{c}(t)$. The uniform distribution of the angles $\psi_{k}$ is achieved, therefore, the agents are distributed uniformly along the time-varying circular formation.

Proof: The stability of the exosystem is analyzed by the composed Lyapunov function $V(\hat{\mathbf{r}}, \psi)=$ $\kappa S(\hat{\mathbf{r}}, \psi)+U(\psi)$. Thanks to the control law (12) then, $\dot{V}(\hat{\mathbf{r}}, \psi)=-\sum_{k=1}^{N}\left(\kappa \omega_{0} \hat{\mathbf{r}}_{k}^{T} \dot{\hat{\mathbf{r}}}_{k}+\frac{\partial U}{\partial \psi_{k}}\right)^{2} \leq 0$ and thus, applying the LaSalle Invariance Principle the exosystem converges to a fixed circular motion with the phase arrangement defined by a critical point of $U(\psi)$. Following the same tracking control design presented in Theorem 1 the control law (8) is obtained and thus $\dot{\mathbf{r}}_{k} \rightarrow \dot{\hat{\mathbf{r}}}_{k}+\dot{\mathbf{c}}, \forall k$ exponentially. It is important to enhance that thanks to this translation control law all the vehicles converge independently to the same circular motion tracking the time-varying center c. Therefore, after some time $t_{f}$ all the agents describe the same time-varying circular motion. Due to the geometric properties of the circle, the distance between two adjacent agents can be expressed as $\left\|\mathbf{r}_{k}-\mathbf{r}_{j}\right\|=2 R \sin \frac{\phi_{k j}}{2}$ where $\phi_{k j}$ is the angular distance between agents $k, j$, see Fig. 2 . If the agents are not uniformly distributed along the circle then, the angular distance between at least two adjacent agents satisfies $\phi_{k j}<2 \pi / N$. Therefore, due to condition (11), the distance between those agents satisfies $\left\|\mathbf{r}_{k}-\mathbf{r}_{j}\right\|<2 R \sin \frac{\pi}{N}<\rho$. Consequently, thanks to the distance-dependent communication graph defined by the Laplacian matrix (10), the agents are connected. The gradient term $-\frac{\partial U}{\partial \psi_{k}}$ enforces agent $k$ to move away from its adjacent agents until the communication between them is broken, i.e., $\left\|\mathbf{r}_{k}-\mathbf{r}_{j}\right\| \geq \rho$. Due to condition (11), the previous 
inequality is equivalent to $\left\|\mathbf{r}_{k}-\mathbf{r}_{j}\right\| \geq 2 R \sin \frac{\pi}{N}$ and then $\phi_{k j} \geq \frac{2 \pi}{N}$. We can repeat the same reasoning for each pair of adjacent agents in the circle and we conclude that the agents will be connected in a circulant graph (each agent will have at least two neighborgs). In this situation, we study the minimum of $U(\psi)$. This potential function can be rewritten as

$$
U(\psi)=\frac{K}{N} \sum_{m=1}^{\lfloor N / 2\rfloor} \frac{1}{2 m^{2}} \sum_{k=1}^{N} U_{m}^{k}
$$

where $U_{m}^{k}=\sum_{j \in \mathcal{N}_{k}} 1+\cos m \psi_{k j} \geq 0$, which is discontinuous because of the definition of the Laplacian matrix $\mathbf{L}$. In order to reach its minimum, the potential function enforces convergence to the splay state because $\psi_{k j} \rightarrow \pi, \forall j \in \mathcal{N}_{k}$. According to [7], the other critical points of $U(\psi)$ for a fixed circulant graph imply at least two agents in the same position. Mathematically, if two agents are in the same position then $\phi_{k j}=0 \Rightarrow \psi_{k j}=0$ and due to the distance-dependent graph that implies a positive contribution $1+\cos \psi_{k j}=2$ to $U_{m}$. Therefore, $U(\psi)$ increases instead to decrease towards the minimum. In conclusion, considering a distance-dependent communication graph, the only minimum of the potential function is the splay state, because all of the other configurations imply two agents in the same position.

\section{Elastic Formation CONTROL}

This section focuses on the design of a general formation control law using a new framework. The objective is to extend the previous translation control law employing affine transformations. In the sequel, a new general formation control law is proposed to stabilize a group of vehicles to a richer class of timevarying configurations, not only circular. The shape of the formation is defined by a general transformation matrix.

\section{A. Affine Transformations}

The three affine transformations are the translation, the scaling and the rotation. To express these affine transformations in a matrix way the homogeneous coordinates are introduced, see [18]. The homogeneous coordinates of a vector $\mathbf{z} \in \mathbb{R}^{2}$ can simply be defined as the new vector $\mathbf{z}^{h}=\left(z_{x}, z_{y}, 1\right)^{T}$. The affine transformations, translation, scaling and rotation are respectively defined by the following matrices:

$$
\mathbf{T}_{c}=\left[\begin{array}{ccc}
1 & 0 & c_{x} \\
0 & 1 & c_{y} \\
0 & 0 & 1
\end{array}\right] \quad \mathbf{S}=\left[\begin{array}{ccc}
s_{x} & 0 & 0 \\
0 & s_{y} & 0 \\
0 & 0 & 1
\end{array}\right] \quad \mathbf{R}_{\phi}=\left[\begin{array}{ccc}
\cos \phi & -\sin \phi & 0 \\
\sin \phi & \cos \phi & 0 \\
0 & 0 & 1
\end{array}\right]
$$


where $s_{x}, s_{y}>0$. The translation in the plane of $\mathbf{z}$ by a vector $\mathbf{c}=\left(c_{x}, c_{y}\right)^{T}$ can be expressed in a matrix multiplication of the form $\mathbf{z}^{\prime}=\mathbf{T}_{c} \mathbf{z}^{h}$ where $\mathbf{z}^{\prime}$ is expressed in homogeneous coordinates. A nonuniform scaling is a transformation such that $\mathbf{z}^{\prime}=\mathbf{S} \mathbf{z}^{h}$. A rotation through an angle $\phi$ counterclockwise around the origin can be written as $\mathbf{z}^{\prime}=\mathbf{R}_{\phi} \mathbf{z}^{h}$.

\section{B. Definition of Elastic Formation}

Roughly speaking, the main idea is to deform the unit circle using the affine transformations in order to obtain a desired elastic formation. In this context, the unit circle $\mathcal{C}_{0}$ is defined as a circumference centered at the origin of the frame and with unit radius. A general sequence of affine transformations is defined as follows:

$$
\mathbf{G}=\prod_{i=1}^{n} \mathbf{A}_{i} \quad \mathbf{A} \in\left\{\mathbf{S}, \mathbf{R}_{\phi}, \mathbf{T}_{c}\right\}
$$

where $n$ is the number of transformations applied to $\mathcal{C}_{0}$. Note that the combination of affine transformations is not commutative. Thus, the order of the sequence of matrices in (13) is important to characterize a particular formation. These affine transformations are invertible, therefore the inverse matrix of $\mathbf{G}$

exists and is denoted by $\mathbf{G}^{-1}$. Thanks to previous definitions, $\mathbf{G}$ is differentiable if its parameters are differentiable.

Definition 1 An elastic formation $\mathcal{F}$ is a curve which results of applying a sequence of affine transformations $\boldsymbol{G}$ defined by (13) to the unit circle $\mathcal{C}_{0}$ such that, $\mathcal{F}=\boldsymbol{G} \circ \mathcal{C}_{0}$.

The elastic formation can be time-varying if at least one element of the transformation matrices is timevarying. The final formation depends on the sequence used to define $\mathbf{G}$. The term elastic denote the capability of the formation to move and change its shape in order, for instance, to avoid an obstacle (see Fig. 3), to delimit a polluted region, or to avoid unnecessary energy waste.

\section{Control Design for Elastic Formations}

In the sequel, in order to apply the affine transformations, all the vectors are expressed in homogeneous coordinates. The position vector of the agent $k$ in homogeneous coordinates is now defined as $\mathbf{r}_{k}=$ $\left(x_{k}, y_{k}, 1\right)^{T}$. Using previous definitions of elastic formation and the general transformation matrix, the following theorem presents the main result of this paper.

Theorem 2 Let $\boldsymbol{G}$ be a twice differentiable matrix function with bounded derivatives resulting of a sequence of affine transformations as defined in (13) and $\mathcal{F}=\boldsymbol{G} \circ \mathcal{C}_{0}$ be the desired elastic formation. 
Let $\omega_{0} \neq 0, \kappa>0, \alpha>0, \beta \geq 2 \sqrt{\alpha}$ and $K>0$ be five control parameters and the following condition is satisfied:

$$
\left\|\dot{\boldsymbol{G}}(t) \hat{\boldsymbol{r}}_{k}\right\| \neq\left|\omega_{0}\right|\left\|\boldsymbol{G}(t) \hat{\boldsymbol{r}}_{k}\right\| \quad \forall t .
$$

Let $\mathcal{G}(t)$ be a communication graph and $\mathbf{L}(t)$ be its corresponding Laplacian matrix. Then, for all initial conditions $\boldsymbol{r}(0), \theta(0)$, the control law:

$$
\begin{aligned}
& \dot{v}_{k}=-\beta v_{k}+\frac{\hat{u}_{k}}{v_{k}} \dot{\boldsymbol{r}}_{k}^{T} \boldsymbol{G} \boldsymbol{R}_{\frac{\pi}{2}} \dot{\hat{\boldsymbol{r}}}_{k}+\frac{\dot{\boldsymbol{r}}_{k}^{T}}{v_{k}}\left[\ddot{\boldsymbol{G}} \hat{\boldsymbol{r}}_{k}+2 \dot{\boldsymbol{G}} \dot{\hat{\boldsymbol{r}}}_{k}+\beta\left(\dot{\boldsymbol{G}} \hat{\boldsymbol{r}}_{k}+\boldsymbol{G} \dot{\hat{\boldsymbol{r}}}_{k}\right)+\alpha\left(\boldsymbol{G} \hat{\boldsymbol{r}}_{k}-\boldsymbol{r}_{k}\right)\right] \\
& u_{k}=\frac{\hat{u}_{k}}{v_{k}^{2}} \dot{\boldsymbol{r}}_{k}^{T} \boldsymbol{R}_{\frac{\pi}{2}}^{T} \boldsymbol{G} \boldsymbol{R}_{\frac{\pi}{2}} \dot{\hat{\boldsymbol{r}}}_{k}+\frac{\dot{\boldsymbol{r}}_{k}^{T} \boldsymbol{R}_{\frac{\pi}{2}}^{T}}{v_{k}^{2}}\left[\ddot{\boldsymbol{G}} \hat{\boldsymbol{r}}_{k}+2 \dot{\boldsymbol{G}} \dot{\hat{\boldsymbol{r}}}_{k}+\beta\left(\dot{\boldsymbol{G}} \hat{\boldsymbol{r}}_{k}+\boldsymbol{G} \dot{\hat{\boldsymbol{r}}}_{k}\right)+\alpha\left(\boldsymbol{G} \hat{\boldsymbol{r}}_{k}-\boldsymbol{r}_{k}\right)\right]
\end{aligned}
$$

where $\dot{\hat{\boldsymbol{r}}}_{k}$ and $\hat{u}_{k}$ are defined by (2) with $v_{0}=\left|\omega_{0}\right|$ and (12) respectively, makes all the agents defined by (1) converge to the elastic formation defined by $\mathcal{F}$. Moreover, the uniform distribution of the angles $\psi_{k}$ along $\mathcal{C}_{0}$ is achieved. Therefore, the agents are distributed along the formation $\mathcal{F}$, taking into account the transformation $\boldsymbol{G}$.

Proof: The proof follows the same steps that in Theorem 1 taking into account the formulation based on homogeneous coordinates. The convergence of the exosystem (2) where $\hat{\mathbf{r}}_{k}=\left(\hat{x}_{k}, \hat{y}_{k}, 1\right)^{T}$ is expressed in homogeneous coordinates, to a uniform distributed circular formation is proved in Corrollary 1. Then, the same tracking control design is proposed to enforce the error defined by (4) to converge to zero. Imposing the error dynamics as previously, i.e., $\ddot{\varepsilon}_{k}=-\beta \dot{\varepsilon}_{k}-\alpha \varepsilon_{k}$, the control inputs (15) are determined since:

$$
\begin{aligned}
\ddot{\varepsilon}_{k} & =\ddot{\mathbf{r}}_{k}-\mathbf{G} \ddot{\hat{\mathbf{r}}}_{k}-2 \dot{\mathbf{G}} \dot{\hat{\mathbf{r}}}_{k}-\ddot{\mathbf{G}} \hat{\mathbf{r}}_{k} \\
-\beta \dot{\varepsilon}_{k}-\alpha \varepsilon_{k} & =\frac{\dot{v}_{k}}{v_{k}} \dot{\mathbf{r}}_{k}+u_{k} \mathbf{R}_{\frac{\pi}{2}} \dot{\mathbf{r}}_{k}-\hat{u}_{k} \mathbf{G R} \frac{\pi}{2} \dot{\hat{\mathbf{r}}}_{k}-2 \dot{\mathbf{G}} \dot{\hat{\mathbf{r}}}_{k}-\ddot{\mathbf{G}} \hat{\mathbf{r}}_{k} .
\end{aligned}
$$

Therefore, $\mathbf{r}_{k} \rightarrow \mathbf{G} \hat{\mathbf{r}}_{k}$ when $t \rightarrow \infty$ and the closed-loop dynamics of system (1) converges to the desired elastic formation $\mathcal{F}$ because for all $k=1, \ldots, N$ :

$$
\dot{\mathbf{r}}_{k}=\underbrace{\omega_{0} \mathbf{G} \mathbf{R}_{\frac{\pi}{2}} \mathbf{G}^{-1} \overline{\mathbf{r}}_{k}}_{\text {transformation of the unit circle }}+\underbrace{\dot{\mathbf{G}} \mathbf{G}^{-1} \mathbf{r}_{k}}_{\text {time-varying motion }} .
$$

In this situation, the singular point $v_{k}=\left\|\dot{\mathbf{r}}_{k}\right\|=0$ can be rewritten as $\omega_{0} \mathbf{G} \mathbf{R}_{\frac{\pi}{2}} \hat{\mathbf{r}}_{k}=-\dot{\mathbf{G}} \hat{\mathbf{r}}_{k}$. If condition (14) is satisfied then $\left\|\dot{\mathbf{G}}(t) \hat{\mathbf{r}}_{k}\right\| \neq\left\|\omega_{0} \mathbf{G}(t) \mathbf{R}_{\frac{\pi}{2}} \hat{\mathbf{r}}_{k}\right\|, \forall t$. Therefore, the singular point $v_{k}=0$ is avoided and the control inputs (15) are respectively obtained.

Unlike in our previous work [19], this control law is robust to uncertainties in the initial conditions of the agents' states. 


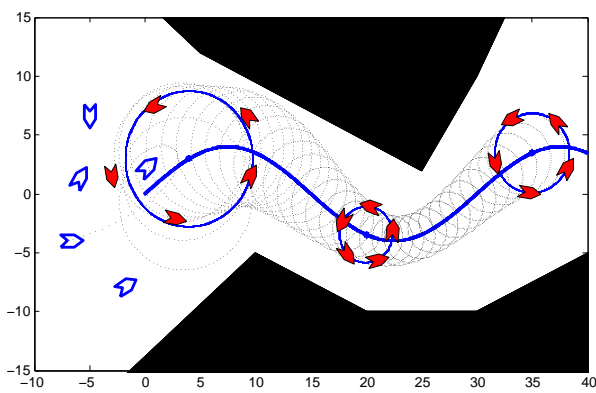

Fig. 3. Simulation of five agents governed by control law (15) with $\mathbf{G}(t)=\mathbf{T}_{c(t)} \mathbf{S}_{R(t)}$. The figure shows four snapshots: the initial conditions (blue void agents), a transient state at $t=40 \mathrm{~s}$ and the stable uniformly distributed circular formation at $t=200 s$ and $t=350 s$.

\section{Simulation Results}

The new formulation presented in this paper makes possible the combination of several transformations to define a complex time-varying formation in a compact and elegant manner. A possible application is a combined motion in which a circular formation with time-varying radius tracks a time-varying center. In this case the transformation matrix defined by (13) becomes $\mathbf{G}(t)=\mathbf{T}_{c(t)} \mathbf{S}_{R(t)}$. Applying Theorem 2, the agents converge to a circular formation which follows the time-varying parameters of the transformation $\mathbf{G}(t)$.

Fig. 3 shows the simulation of five agents governed by (15) where $\mathbf{G}(t)=\mathbf{T}_{c(t)} \mathbf{S}_{R(t)}$. The control law parameters are $\omega_{0}=\kappa=0.6, \alpha=1, \beta=2$ and $K=0.1$. The time-varying reference of the radius is $R(t)=4+2 \cos \frac{2 \pi}{500} t$ and the reference tracked by the center corresponds to $\mathbf{c}(t)=\left(\frac{1}{10} t, 3 \sin \frac{2 \pi}{300} t\right)^{T}$. The agents converge to the time-varying circular formation for any random initial conditions (position and heading of the agents) represented in the figure by the blue void agents. The trajectory of one agent is represented by the blue dotted line. The communication radius considered here $\rho=8$ satisfies the geometrical condition $\rho>2 R_{\max } \sin \frac{\pi}{N}$ from Theorem 2, where $R_{\max }=6$ is the up-bound of the reference of the radius. Consequently, the agents are uniformly distributed along the time-varying circular formation. Videos showing more simulations are accessible through Web ${ }^{1}$.

${ }^{1}$ Simulations are accessible in https://sites.google.com/site/lbrinonarranz/videos 


\section{CONCLUSIONS}

This paper presents a new control strategy to stabilize a group of vehicles to time-varying formations. The main idea is to transform the circular trajectories of a stable autonomous exosystem and to enforce the multi-agent system to track these transformed trajectories. Firstly, we presented a simple case dealing with the translation of a circular motion. In this case, all the agents converge to a circular motion whose center tracks a given time-varying reference. Then, a new framework based on affine transformations (translation, rotation and scaling) is proposed to stabilize the fleet to more complex time-varying formations, not only circular. The main advantage of this approach is that the desired formation is parametrized by a few number of parameters. Moreover, a cooperative term is included to uniformly distribute the agents along the formation taking into account the communication topology between agents.

Future works will focus on designing a general time-varying formation control law in a more cooperative way, using only relative information between agents. Another research direction copes with extending this control strategy including some additional constraints such as the presence of currents and adding potential terms to ensure obstacle avoidance.

\section{ACKNOWLEDGMENTS}

We would like to thank Rodolphe Sepulchre for his useful comments and technical remarks.

\section{REFERENCES}

[1] R. Olfati-Saber and R. M. Murray, "Consensus problems in networks of agents with switching topology and time-delays," IEEE Trans. on Automatic Control, vol. 49, pp. 1520-1533, 2004.

[2] R. Olfati-Saber, J. A. Fax, and R. M. Murray, "Consensus and cooperation in networked multi-agent systems," Proc. IEEE, vol. 95, pp. 215-233, 2007.

[3] N. E. Leonard, D. A. Paley, F. Lekien, R. Sepulchre, D. M. Frantatoni, and R. E. Davis, "Collective motion, sensor networks and ocean sampling," Proc. IEEE, vol. 95, pp. 48-74, 2007.

[4] B. J. Moore and C. Canudas-de-Wit, "Source seeking via collaborative measurements by a circular formation of agents," in Proc. 2010 IEEE American Control Conf., Baltimore, USA, pp. 6417-6422, 2010.

[5] R. L. Raffard, C. J. Tomlin, and S. P. Boyd, "Distributed optimization for cooperative agents: Application to formation flight," in Proc. 43rd IEEE Conf. on Decision and Control, Paradise Islands, The Bahamas, pp. 2453-2459, 2004.

[6] S. Martínez, J. Cortés, and F. Bullo, "Motion coordination with distributed information," IEEE Control Systems Magazine, vol. 27, pp. 75-88, 2007.

[7] R. Sepulchre, D. A. Paley, and N. E. Leonard, "Stabilization of planar collective motion with limited communication," IEEE Trans. on Automatic Control, vol. 53, pp. 706-719, 2008.

[8] S. Hernandez and D. A. Paley, "Three-dimensional motion coordination in a time-invariant flowfield," in Proc. 48th IEEE Conf. on Decision and Control, Shanghai, China, pp. 7043-7048, 2009. 
[9] D. A. Paley, N. E. Leonard, and R. Sepulchre, "Stabilization of symmetric formations to motion around convex loops," Systems \& Control Letters, vol. 57, pp. 209-215, 2008.

[10] P. Ögren, E. Fiorelli, and N. E. Leonard, "Cooperative control of mobile sensor networks: Adaptive gradient climbing in a distributed environment," IEEE Trans. on Automatic Control, vol. 49, pp. 1292-1302, 2004.

[11] D. J. Klein and K. A. Morgansen, "Controlled collective motion for trajectory tracking," in Proc. 2006 American Control Conf., Minneapolis, MN, USA, pp. 5269-5275, 2006.

[12] Y. Lan, Z. Lin, M. Cao, and G. Yan, "A distributed reconfigurable control law for escorting and patrolling missions using teams of unicycles," in Proc. 49th IEEE Conf. on Decision and Control, Atlanta, GA, USA, pp. 5456-5461, 2010.

[13] V. M. Goncalves, L. C. A. Pimenta, C. A. Maia, B. C. O. Dutra, and G. A. S. Pereira, "Vector fields for robot navigation along time-varying curves in n-dimensions," IEEE Trans. on Robotics, vol. 29, pp. 647-659, 2010.

[14] E. W. Frew and D. Lawrence, "Tracking expanding star curves using guidance vector fields," in Proc. 2012 American Control Conf., Montréal, Canada, pp. 1749-1754, 2012.

[15] D. A. Paley, N. E. Leonard, and R. Sepulchre, "Oscillator models and collective motion: Splay state stabilization of self-propelled particles," in Proc. 44th IEEE Conf. on Decision and Control, Seville, Spain, pp. 3935-3940, 2005.

[16] L. Briñón-Arranz, A. Seuret, and C. Canudas-de-Wit, "Translation control of a fleet circular formation of AUVs under finite communication range," in Proc. 48th IEEE Conf. on Decision and Control, Shanghai, China, pp. 8345-8350, 2009.

[17] J. Cortés, S. Martínez, and F. Bullo, "Robust rendezvous for mobile autonomous agents via proximity graphs in arbitrary dimensions," IEEE Trans. on Automatic Control, vol. 51, pp. 1289-1298, 2006.

[18] J. D. Foley, A. van Dam, S. K. Feiner, J. F. Hughes, and R. L. Phillips, Introduction to Computer Graphics. Addison-Wesley Publishing Co., 1994.

[19] L. Briñón-Arranz, A. Seuret, and C. Canudas-de-Wit, "Elastic formation control based on affine transformations," in Proc. 2011 American Control Conf., San Francisco, CA, USA, pp. 3984-3989, 2011. 